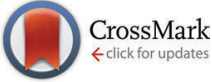

Cite this: Phys. Chem. Chem. Phys., 2016, 18, 8545

Received 24th November 2015, Accepted 24th February 2016

DOI: $10.1039 / c 5 c p 07251 d$

www.rsc.org/pccp

\title{
Director orientations in lyotropic liquid crystals: diffusion MRI mapping of the Saupe order tensor
}

\author{
Daniel Topgaard
}

\begin{abstract}
The macroscopic physical properties of a liquid crystalline material depend on both the properties of the individual crystallites and the details of their spatial arrangement. We propose a diffusion MRI method to estimate the director orientations of a lyotropic liquid crystal as a spatially resolved field of Saupe order tensors. The method relies on varying the shape of the diffusion-encoding tensor to disentangle the effects of voxel-scale director orientational order and the local diffusion anisotropy of the solvent. Proof-of-concept experiments are performed on water in lamellar and reverse hexagonal liquid crystalline systems with intricate patterns of director orientations.
\end{abstract}

\section{Introduction}

Anisotropic assemblies of amphiphilic molecules in aqueous media occur in a wide range of materials: from lyotropic liquid crystals $^{1-3}$ to brain tissue. ${ }^{4}$ Locally, the amphiphiles have a preferred orientation with respect to a unit vector known as the director ${ }^{5}$ and a liquid crystalline domain can be defined as a region of space in which the director has a constant orientation. Material properties, such as optical birefringence, electrical conductivity, and molecular diffusivity, are determined by the local properties within a single domain as well as the spatial pattern of director orientations, the latter being possible to influence by temperature cycling, shear, magnetic fields, or the presence of solid surfaces. ${ }^{6-27}$ For technical applications of liquid crystals in drug delivery ${ }^{28,29}$ or templating of inorganic materials, ${ }^{30,31}$ it is desirable to control the domain sizes and orientations.

The orientational order of an ensemble of unit vectors is often expressed as the Saupe order tensor $\mathbf{S}$ with elements $S_{i j}$ defined by ${ }^{5,32-34}$

$$
S_{i j}=\frac{1}{2}\left\langle 3 l_{i} l_{j}-k_{i j}\right\rangle
$$

where $i, j \in\{x, y, z\},\langle\cdot\rangle$ denotes an ensemble average, $k_{i j}$ is the Kronecker delta, and $l_{i}$ are the directional cosines of the vectors in the lab frame $x y z$. The order tensor contains five independent elements and is often parameterized with the principal order parameter $S_{Z Z}$, the asymmetry parameter $\eta$, and three Euler angles describing the orientation of the principal axis system $X Y Z$ with respect to the lab frame. In the Landau-de Gennes theory of nematic liquid crystals, ${ }^{5,34}$ and its extension

Division of Physical Chemistry, Department of Chemistry, Lund University, Lund, Sweden. E-mail: daniel.topgaard@fkem1.lu.se to lyotropic nematic liquid crystals, ${ }^{35,36}$ the free energy density at the position $\mathbf{r}$ is determined by the local order tensor $\mathbf{S}(\mathbf{r})$. Hence, the results of mean-field calculations of the structure of liquid crystals are often visualized as spatially resolved fields of order tensors. ${ }^{37-39}$ An experimental method capable of mapping such tensor fields would enable critical testing of the results of theoretical calculations and allow for detailed characterization of liquid crystals for technical applications.

The structure of a lyotropic liquid crystal is imprinted in the orientational order and translational diffusion of the water located in the nanometer-scale gaps between the amphiphile aggregates. The orientational order of the water can be detected with ${ }^{2} \mathrm{H}$ nuclear magnetic resonance (NMR) spectroscopy as a quadrupolar splitting of the ${ }^{2} \mathrm{H}_{2} \mathrm{O}$ resonance line, ${ }^{40-42}$ while the structural anisotropy of the liquid crystal gives rise to a directional dependence of the water self-diffusion coefficient as observed with diffusion $\mathrm{NMR}^{13,43-48}$ and magnetic resonance imaging (MRI) ${ }^{24,27,49,50}$ Despite the fact that these NMR and MRI methods have been extensively used for investigating macroscopic domain alignment in liquid crystals, ${ }^{8,9,14,15,18,19,21,23,24,26,27,40,44,49-55}$ there are so far no reported studies where the full order tensor has been mapped with spatial resolution. In principle, such tensor maps could be obtained by acquiring spatially resolved ${ }^{2} \mathrm{H}$ spectra ${ }^{24,27,55-57}$ for multiple orientations of the main magnetic field. ${ }^{58}$ Unfortunately, such an experimental approach would require combinations of NMR hardware that are exceedingly rare.

Here, we introduce a diffusion MRI method for mapping director order tensors in lyotropic liquid crystals. The new method builds on conventional diffusion tensor imaging (DTI), ${ }^{59}$ which yields the average diffusion tensor $\langle\mathbf{D}\rangle$ for each spatially resolved volume element, "voxel", of the image, and our recent method for quantifying the microscopic diffusion tensor $\mathbf{D}$ within a single liquid crystalline domain of a polydomain sample. ${ }^{60}$ 
The latter method relies on an acquisition protocol wherein not only the magnitude and direction of the diffusion-encoding is varied, as in conventional DTI, but also the shape of the axisymmetric diffusion-encoding tensor b. ${ }^{60-64}$ In the theory section, we present a detailed derivation of the relation between the tensors $\langle\mathbf{D}\rangle, \mathbf{D}$, and $\mathbf{S}$, parts of which have previously appeared in the literature, ${ }^{27,50,65-69}$ and describe how order tensor fields can be calculated from independently measured maps of $\langle\mathbf{D}\rangle$ and $\mathbf{D}$. We demonstrate the new method by proofof-principle experiments on lamellar and reverse hexagonal lyotropic liquid crystals with a range of director orientation distributions. The experiments are carried out with a slightly modified version of the diffusion MRI pulse sequence introduced by Lasič et al..$^{50}$ and subsequently used for human in vivo studies in a series of recent publications. ${ }^{62,64,69}$ We also elaborate on the procedure for generating axisymmetric diffusion-encoding with smoothly modulated waveforms for the time-dependent magnetic field gradients. ${ }^{70}$

\section{Theoretical considerations}

The theory section includes derivations of the expressions for estimating the tensors $\langle\mathbf{D}\rangle, \mathbf{D}$, and $\mathbf{S}$ from experimental data, as well as a summary of the principles of axisymmetric diffusion-encoding

$$
\mathbf{D}=D_{\text {iso }}\left[\mathbf{I}+2 D_{\Delta} \cdot \frac{1}{2}\left(\begin{array}{c}
3 \cos ^{2} \phi \sin ^{2} \theta-1 \\
3 \sin \phi \cos \phi \sin ^{2} \theta \\
3 \cos \phi \sin \theta \cos \theta
\end{array}\right.\right.
$$

as recently introduced by Eriksson et al. ${ }^{60}$ Readers mainly interested in the experimental demonstration of the new approach may wish to go directly to the Results and discussion section, and simply note that the key equations for data evaluation can be found in eqn (12), (19), and (29).

\section{Diffusion and order tensors}

In its principal axis system (PAS), a microscopic diffusion tensor $\mathbf{D}$ can be written as

$$
\mathbf{D}^{\mathrm{PAS}}=\left(\begin{array}{ccc}
D_{X X} & 0 & 0 \\
0 & D_{Y Y} & 0 \\
0 & 0 & D_{Z Z}
\end{array}\right)
$$

with the eigenvalues ordered as $\left(D_{Z Z}-D_{\text {iso }}\right) \geq\left(D_{X X}-D_{\text {iso }}\right) \geq$ $\left(D_{Y Y}-D_{\text {iso }}\right)$, where $D_{\text {iso }}$ is the isotropic average of the eigenvalues:

$$
D_{\text {iso }}=\frac{1}{3}\left(D_{X X}+D_{Y Y}+D_{Z Z}\right)
$$

Defining the diffusion tensor anisotropy $D_{\Delta}$ and asymmetry $D_{\eta}$ as $^{44,60,67}$

$$
D_{\Delta}=\frac{1}{3 D_{\text {iso }}}\left(D_{Z Z}-\frac{D_{Y Y}+D_{X X}}{2}\right) \text { and } D_{\eta}=\frac{D_{Y Y}-D_{X X}}{2 D_{\text {iso }} D_{\Delta}} \text {, }
$$

Eqn (2) can be rewritten as

$$
\begin{aligned}
\mathbf{D}^{\text {PAS }}=D_{\text {iso }} & \left\{\left(\begin{array}{ccc}
1 & 0 & 0 \\
0 & 1 & 0 \\
0 & 0 & 1
\end{array}\right)\right. \\
& \left.+D_{\Delta}\left[\left(\begin{array}{ccc}
-1 & 0 & 0 \\
0 & -1 & 0 \\
0 & 0 & 2
\end{array}\right)+D_{\eta}\left(\begin{array}{ccc}
-1 & 0 & 0 \\
0 & 1 & 0 \\
0 & 0 & 0
\end{array}\right)\right]\right\},
\end{aligned}
$$

which is reduced to

$$
\mathbf{D}^{\mathrm{PAS}}=D_{\text {iso }}\left[\mathbf{I}+2 D_{\Delta}\left(\begin{array}{ccc}
-1 / 2 & 0 & 0 \\
0 & -1 / 2 & 0 \\
0 & 0 & 1
\end{array}\right)\right]
$$

if the diffusion tensor is axisymmetric $\left(D_{\eta}=0\right)$. In eqn (6), I is the identity matrix. While $D_{\text {iso }}$ corresponds to the "size" of the tensor, the value of $D_{\Delta}$ reports on its "shape", covering the range from $-1 / 2$ (planar) to 0 (spherical) and +1 (linear). ${ }^{60}$

If the eigenframe $X Y Z$ of the axisymmetric tensor $\mathbf{D}^{\mathrm{PAS}}$ is initially aligned with the lab frame $x y z$, rotation through the polar and azimuthal angles $\theta$ and $\phi$ yields a lab-frame tensor $\mathbf{D}$ given by

$\left.\left.\begin{array}{cc}3 \sin \phi \cos \phi \sin ^{2} \theta & 3 \cos \phi \sin \theta \cos \theta \\ 3 \sin ^{2} \phi \sin ^{2} \theta-1 & 3 \sin \phi \sin \theta \cos \theta \\ 3 \sin \phi \sin \theta \cos \theta & 3 \cos ^{2} \theta-1\end{array}\right)\right]$.

Replacing the trigonometric expressions in eqn (7) with the directional cosines

$$
\begin{gathered}
l_{x}=\cos \phi \sin \theta \\
l_{y}=\sin \phi \sin \theta \\
l_{z}=\cos \theta
\end{gathered}
$$

gives

$$
\mathbf{D}=D_{\text {iso }}\left[\mathbf{I}+2 D_{\Delta} \cdot \frac{1}{2}\left(\begin{array}{ccc}
3 l_{x}^{2}-1 & 3 l_{x} l_{y} & 3 l_{x} l_{z} \\
3 l_{x} l_{y} & 3 l_{y}^{2}-1 & 3 l_{y} l_{z} \\
3 l_{x} l_{z} & 3 l_{y} l_{z} & 3 l_{z}^{2}-1
\end{array}\right)\right] .
$$

The terms with directional cosines can be recognized from the definition of the Saupe order tensor $\mathbf{S}$ in eqn (1), which in matrix form can be written as

$$
\mathbf{S}=\frac{1}{2}\left(\begin{array}{ccc}
\left\langle 3 l_{x}^{2}-1\right\rangle & \left\langle 3 l_{x} l_{y}\right\rangle & \left\langle 3 l_{x} l_{z}\right\rangle \\
\left\langle 3 l_{x} l_{y}\right\rangle & \left\langle 3 l_{y}^{2}-1\right\rangle & \left\langle 3 l_{y} l_{z}\right\rangle \\
\left\langle 3 l_{x} l_{z}\right\rangle & \left\langle 3 l_{y} l_{z}\right\rangle & \left\langle 3 l_{z}^{2}-1\right\rangle
\end{array}\right)
$$

The principal order parameter $S_{Z Z}$ is defined as the eigenvalue of $\mathbf{S}$ with the largest magnitude. ${ }^{33}$ The values of $S_{Z Z}$ cover the range from $-1 / 2$ to +1 . Perfect alignment in a single direction 
corresponds to $S_{Z Z}=1$, while random orientations in a plane perpendicular to the director gives $S_{Z Z}=-1 / 2$. The value $S_{Z Z}=0$ indicates completely random orientations in $3 \mathrm{D}$ space, but could also result from other, more exotic, orientation distributions, e.g. , three orthogonal directions with equal probability or random orientations on a cone with aperture $109.4^{\circ}$.

Assuming that there is no molecular exchange between the domains on the 10-100 ms time-scale defined by the diffusion NMR experiment, then $\langle\mathbf{D}\rangle$ is simply the population-weighted average of the domain tensors D. ${ }^{65}$ For an ensemble of tensors with the same size $D_{\text {iso }}$ and shape $D_{\Delta}$, but different orientations $(\theta, \phi)$, application of ensemble averaging to both sides of eqn (9) yields

$$
\langle\mathbf{D}\rangle=D_{\text {iso }}\left(\mathbf{I}+2 D_{\Delta} \mathbf{S}\right),
$$

where $\langle\mathbf{D}\rangle$ is the ensemble-average or "voxel-average" diffusion tensor as measured with standard DTI. ${ }^{59}$ Quantitative estimates of $D_{\text {iso }}$ and $D_{\Delta}$ can be obtained with the method of axisymmetric diffusion-encoding introduced by Eriksson et al. ${ }^{60}$ and described below. Once $\langle\mathbf{D}\rangle, D_{\text {iso }}$ and $D_{\Delta}$ have been determined, $\mathbf{S}$ can be calculated through element-by-element inversion of eqn (11):

$$
S_{i j}=\frac{1}{2 D_{\Delta}} \cdot\left(\frac{\left\langle D_{i j}\right\rangle}{D_{\text {iso }}}-k_{i j}\right) .
$$

From eqn (11) follows that the anisotropy $\lambda_{\Delta}$ of the average tensor $\langle\mathbf{D}\rangle$ is given by ${ }^{67}$

$$
\lambda_{\Delta}=S_{Z Z} D_{\Delta} .
$$

Maximal macroscopic anisotropy $\left(\lambda_{\Delta}=1\right)$ requires that both the microscopic anisotropy $D_{\Delta}$ and the principal order parameter $S_{Z Z}$ equal 1. Conversely, a planar macroscopic tensor $\left(\lambda_{\Delta}=-1 / 2\right)$ could result from either perfect alignment of planar microscopic tensors $\left(S_{Z Z}=1, D_{\Delta}=-1 / 2\right)$ or negative uniaxial alignment of linear microscopic tensors $\left(S_{Z Z}=-1 / 2, D_{\Delta}=1\right)$.

Diffusion tensors are often visualized as ellipsoid ${ }^{59}$ or superquadric $^{71}$ tensor glyphs, where the lengths and directions of the three semi-axes are given by the corresponding tensor eigenvalues and eigenvectors. According to the definition in eqn (1), the tensor $\mathbf{S}$ is traceless and has both positive and negative eigenvalues, which cannot directly be represented as the conventional tensor glyphs. Various approaches for manipulating the order tensor to facilitate visualization can be found in the literature. ${ }^{37-39}$ Here, we define a shifted and rescaled order tensor $\mathbf{S}^{\prime}$ through

$$
\mathbf{S}^{\prime}=\frac{1}{3}(\mathbf{I}+2 \mathbf{S})
$$

The eigenvalues of the symmetric and unit-trace tensor $\mathbf{S}^{\prime}$ are all positive, covering the range from 0 to 1 , and the eigenvectors coincide with the ones for $\langle\mathbf{D}\rangle$. Consequently, the $\mathbf{S}^{\prime}$ and $\langle\mathbf{D}\rangle$ tensor fields can be visualized using the same kind of glyphs or color-code.

\section{NMR diffusion-encoding}

The NMR signal is encoded with information about translational motion by applying a time-dependent magnetic field gradient $\mathbf{G}(t)$ in the time interval $0 \leq t \leq \tau$. The diffusionencoding tensor $\mathbf{b}$ is given by ${ }^{72,73}$

$$
\mathbf{b}=\int_{0}^{\tau} \mathbf{q}(t) \mathbf{q}^{\mathrm{T}}(t) \mathrm{d} t
$$

where

$$
\mathbf{q}(t)=\gamma \int_{0}^{t} \mathbf{G}\left(t^{\prime}\right) \mathrm{d} t^{\prime}
$$

is the time-dependent dephasing vector and $\gamma$ is the magnetogyric ratio of the studied nucleus. The gradient waveform $\mathbf{G}(t)$ obeys the "echo condition" $q(\tau)=0$.

For a sample or volume element comprising an ensemble of microscopic diffusion tensors $\mathbf{D}$, the NMR signal $I(\mathbf{b})$ can be written as $^{72,73}$

$$
I(\mathbf{b})=I_{0}\langle\exp (-\mathbf{b}: \mathbf{D})\rangle
$$

where $I_{0}$ is the signal when $b=0$ and $\mathbf{b}: \mathbf{D}$ is a generalized scalar product defined as

$$
\mathbf{b}: \mathbf{D}=\sum_{i} \sum_{j} b_{i j} D_{i j}
$$

In the limit $b \rightarrow 0$, eqn (19) can be approximated as ${ }^{64}$

$$
I(\mathbf{b})=I_{0} \exp (-\mathbf{b}:\langle\mathbf{D}\rangle),
$$

where $\langle\mathbf{D}\rangle$ is the ensemble-average diffusion tensor. Eqn (19) corresponds to the conventional equation for evaluating DTI data. ${ }^{72,73}$

\section{Parameterization of the b-tensor}

In analogy with the description of the diffusion tensor above, the b-tensor can, in its principal axis system, be expressed as

$$
\mathbf{b}^{\mathrm{PAS}}=\left(\begin{array}{ccc}
b_{X X} & 0 & 0 \\
0 & b_{Y Y} & 0 \\
0 & 0 & b_{Z Z}
\end{array}\right),
$$

where the eigenvalues are ordered according to the convention $\left(b_{Z Z}-b / 3\right) \geq\left(b_{X X}-b / 3\right) \geq\left(b_{Y Y}-b / 3\right)$, and $b$ is the trace of the b-tensor:

$$
b=b_{X X}+b_{Y Y}+b_{Z Z} .
$$

The b-tensor anisotropy $b_{\Delta}$ and asymmetry $b_{\eta}$ are given by ${ }^{60}$

$$
b_{\Delta}=\frac{1}{b}\left(b_{Z Z}-\frac{b_{Y Y}+b_{X X}}{2}\right) \text { and } b_{\eta}=\frac{3}{2} \cdot \frac{b_{Y Y}-b_{X X}}{b b_{\Delta}} .
$$

With the parameterization in eqn (21) and (22), eqn (20) can be recast into

$$
\begin{aligned}
\mathbf{b}^{\text {PAS }}= & \frac{b}{3}\left\{\left(\begin{array}{lll}
1 & 0 & 0 \\
0 & 1 & 0 \\
0 & 0 & 1
\end{array}\right)\right. \\
+b_{\Delta} & {\left.\left[\left(\begin{array}{ccc}
-1 & 0 & 0 \\
0 & -1 & 0 \\
0 & 0 & 2
\end{array}\right)+b_{\eta}\left(\begin{array}{ccc}
-1 & 0 & 0 \\
0 & 1 & 0 \\
0 & 0 & 0
\end{array}\right)\right]\right\} . }
\end{aligned}
$$


Axisymmetric diffusion-encoding corresponds to $b_{\eta}=0$, and a simplified expression for the $\mathbf{b}$-tensor can be written as

$$
\mathbf{b}^{\mathrm{PAS}}=\frac{b}{3}\left[\mathbf{I}+2 b_{\Delta}\left(\begin{array}{ccc}
-1 / 2 & 0 & 0 \\
0 & -1 / 2 & 0 \\
0 & 0 & 1
\end{array}\right)\right] .
$$

For an axisymmetric b-tensor initially aligned with the lab frame, rotation through the polar and azimuthal angles $\Theta$ and $\Phi$ gives the lab-frame $\mathbf{b}$-tensor

and the polar angle $\zeta$ is constant. In eqn (31), $b$ is the trace of the b-tensor, which can be calculated with eqn (15) and (20), or, alternatively, directly from $q(t)$ using $^{75}$

$$
b=\int_{0}^{\tau} q(t)^{2} \mathrm{~d} t .
$$

The angle $\zeta$ determines the b-tensor anisotropy $b_{\Delta}$ according to ${ }^{60}$

$$
b_{\Delta}=P_{2}(\cos \zeta) \text {, }
$$

$$
\mathbf{b}=\frac{b}{3}\left[\mathbf{I}+b_{\Delta}\left(\begin{array}{ccc}
3 \cos ^{2} \Phi \sin ^{2} \Theta-1 & 3 \sin \Phi \cos \Phi \sin ^{2} \Theta & 3 \cos \Phi \sin \Theta \cos \Theta \\
3 \sin \Phi \cos \Phi \sin ^{2} \Theta & 3 \sin ^{2} \Phi \sin ^{2} \Theta-1 & 3 \sin \Phi \sin \Theta \cos \Theta \\
3 \cos \Phi \sin \Theta \cos \Theta & 3 \sin \Phi \sin \Theta \cos \Theta & 3 \cos ^{2} \Theta-1
\end{array}\right)\right] .
$$

\section{Powder-averaged signal}

Inserting the expressions for the axisymmetric tensors $\mathbf{b}$ and D in eqn (7) and (25), respectively, into eqn (18) yields

$$
\mathbf{b}: \mathbf{D}=b D_{\text {iso }}\left[1+2 b_{\Delta} D_{\Delta} P_{2}(\cos \beta)\right],
$$

where

$$
\cos \beta=\cos \Theta \cos \theta+\sin \Theta \sin \theta \cos (\Phi-\phi),
$$

and $P_{2}(x)=\left(3 x^{2}-1\right) / 2$ is the 2 nd Legendre polynomial. For samples comprising randomly oriented microscopic domains, or when powder-averaged signal acquisition is applied, ${ }^{50}$ the probability distribution $P(\beta)$ of the angle $\beta$ is given by

$$
P(\beta)=\frac{1}{2} \sin \beta
$$

in the interval $0 \leq \beta \leq 180^{\circ}$. Using eqn (26) and (28) when evaluating the ensemble average in eqn (17) yields ${ }^{60}$

$$
\begin{aligned}
I\left(b, b_{\Delta}\right)= & I_{0} \exp \left(-b D_{\text {iso }}\right) \\
& \times \frac{\sqrt{\pi}}{2} \frac{\exp \left(b D_{\text {iso }} b_{\Delta} D_{\Delta}\right) \operatorname{erf}\left(\sqrt{3 b D_{\text {iso }} b_{\Delta} D_{\Delta}}\right)}{\sqrt{3 b D_{\text {iso }} b_{\Delta} D_{\Delta}}},
\end{aligned}
$$

where $\operatorname{erf}(x)$ is the error function. Eqn (29) can be used to extract values of $D_{\text {iso }}$ and $D_{\Delta}$ by analyzing the powder-averaged signal $I\left(b, b_{\Delta}\right)$ acquired as a function of $b$ and $b_{\Delta} \cdot{ }^{60}$

\section{Gradient waveforms for axisymmetric diffusion-encoding}

An axially symmetric b-tensor can be obtained by selecting a q-vector trajectory

$$
\mathbf{q}(t)=\left[\begin{array}{c}
q_{X}(t) \\
q_{Y}(t) \\
q_{Z}(t)
\end{array}\right]=q(t)\left[\begin{array}{c}
\cos [\psi(t)] \sin (\zeta) \\
\sin [\psi(t)] \sin (\zeta) \\
\cos (\zeta)
\end{array}\right]
$$

where the q-vector magnitude $q(t)$ and azimuthal angle $\psi(t)$ satisfy the relation ${ }^{70,74}$

$$
\psi(t)=\frac{2 \pi}{b} \int_{0}^{t} q(t)^{2} \mathrm{~d} t
$$

where $P_{2}(x)$ is the 2nd Legendre polynomial as defined below eqn (27). The gradient $\mathbf{G}(t)$ is given by the derivative

$$
\mathbf{G}(t)=\frac{1}{\gamma} \cdot \frac{\mathrm{d}}{\mathrm{d} t} \mathbf{q}(t) .
$$

Explicit gradient waveforms obeying the constraints above can be constructed by selecting an axial waveform $G_{\mathrm{A}}(t)$ from a standard pulsed gradient spin echo experiment with, e.g., rectangular, ramped, sinusoidal, Gaussian, or exponential gradient pulse shapes. ${ }^{76,77}$ The chosen waveform then gives $q(t)$ from

$$
q(t)=\gamma \int_{0}^{t} G_{\mathrm{A}}\left(t^{\prime}\right) \mathrm{d} t^{\prime}
$$

and $b$ and $\psi(t)$ with eqn (31) and (32), respectively. Inserting $q(t)$ and $\psi(t)$ into eqn (30) and (34) yields

$$
\mathbf{G}(t)=\left[\begin{array}{c}
G_{X}(t) \\
G_{Y}(t) \\
G_{Z}(t)
\end{array}\right]=\left[\begin{array}{c}
\operatorname{Re}\left[G_{\mathrm{R}}(t)\right] \sin (\zeta) \\
\operatorname{Im}\left[G_{\mathrm{R}}(t)\right] \sin (\zeta) \\
G_{\mathrm{A}}(t) \cos (\zeta)
\end{array}\right],
$$

where $G_{\mathrm{R}}(t)$ is the complex radial gradient waveform

$$
G_{\mathrm{R}}(t)=\left[G_{\mathrm{A}}(t)+\frac{2 \pi q(t)^{3}}{\gamma b} i\right] \exp [i \psi(t)] .
$$

As shown in Fig. 1(a), axisymmetric diffusion-encoding can be implemented in a diffusion MRI pulse sequence by replacing the conventional rectangular or ramped gradient pulses with the waveform $\mathbf{G}(t){ }^{50,62,64,69}$ The procedure for transforming an axial waveform $G_{\mathrm{A}}(t)$ and a value of $\zeta$ to $\mathbf{G}(t)$ is summarized in Fig. 1(b). First, $G_{\mathrm{A}}(t)$ is converted to $q(t), b$, and $\psi(t)$ using eqn (31), (32), and (35), respectively. Subsequently, these functions and values give the radial waveform $G_{\mathrm{R}}(t)$ via eqn (37). Finally, $\mathbf{G}(t)$ is obtained by combining $G_{\mathrm{A}}(t)$ and $G_{\mathrm{R}}(t)$ with amplitude scaling given by the angle $\zeta$ as described in eqn (36). Under the condition that the $X Y Z$ PAS of the gradients is initially aligned with the $x y z$ lab frame, rotation of $\mathbf{G}(t)$ through the angles $(\Theta, \Phi)$ yields b-tensor elements according to eqn (25) with $b$ and $b_{\Delta}$ being given by $G_{\mathrm{A}}(t)$ and $\zeta$ via eqn (32), (33), and (35). 
(a)

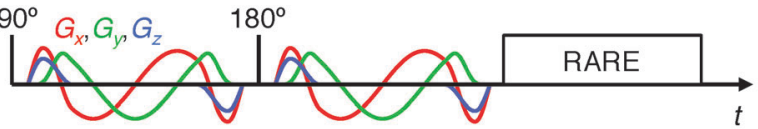

(b)

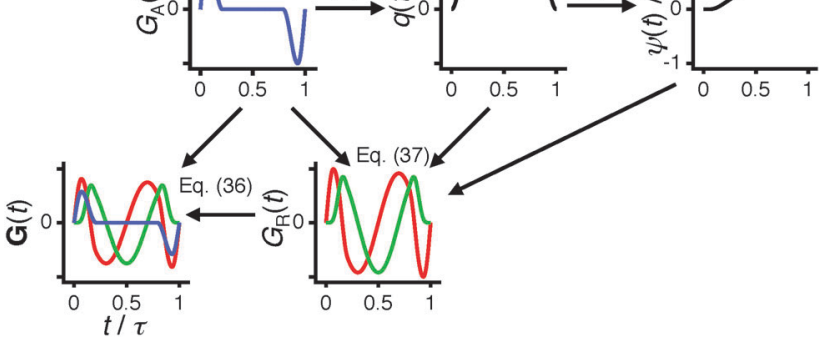

Fig. 1 Axisymmetric diffusion-encoding in diffusion MRI. (a) Pulse sequence with a spin echo $\left(90^{\circ}\right.$ and $180^{\circ}$ RF pulses) preceding RARE image read-out. Identical diffusion-encoding gradient waveforms $\mathbf{G}(t)$ with duration $\tau$ bracket the $180^{\circ}$ pulse. The Cartesian components $G_{x}, G_{y}$, and $G_{z}$ are shown in red, green, and blue, respectively. (b) Flow-scheme for generating $\mathbf{G}(t)$. The panels show the axial and radial waveforms $G_{A}(t)$ and $G_{R}(t)$, and the magnitude $q(t)$ and azimuthal angle $\psi(t)$ of the dephasing vector $\mathbf{q}(t)$. The real and imaginary parts of $G_{R}(t)$ are colored red and green, respectively. The magnitude $b$ and anisotropy $b_{\Delta}$ of the $\mathbf{b}$-tensor are given by $G_{A}(t)$ and the q-vector inclination $\zeta$ via eqn (32), (33) and (35). Rotation of $\mathbf{G}(t)$ through the polar and azimuthal angles $\Theta$ and $\Phi$ results in the corresponding rotation of the $\mathbf{b}$-tensor.

\section{Experimental}

\section{Sample preparation}

The nonionic surfactant penta(ethylene glycol) monotetradecyl ether $\left(\mathrm{C}_{14} \mathrm{E}_{5}\right)$ forms a lamellar phase $\left(\mathrm{L}_{\alpha}\right)$ in water over a wide range of concentrations and temperatures. ${ }^{25}$ The planar geometry of the water compartments in the $\mathrm{L}_{\alpha}$ phase gives rise to a correspondingly planar shape of the microscopic diffusion tensor and a value of $D_{\Delta}$ approaching $-1 / 2 .{ }^{60}$ The phase diagram of sodium 1,4-bis(2-ethylhexoxy)-1,4-dioxobutane-2-sulfonate (AOT)/ 2,2,4-trimethylpentane (isooctane)/water is dominated by a reverse hexagonal phase $\left(\mathrm{H}_{\mathrm{II}}\right)$ at $25{ }^{\circ} \mathrm{C} .{ }^{2}$ The nearly linear shape of the water compartments in the $\mathrm{H}_{\text {II }}$ phase is mirrored in the linear shape of the microscopic diffusion tensors and values of $D_{\Delta}$ near $+1 .^{60,63}$

The liquid crystals were made with water containing $90 \mathrm{wt} \%$ ${ }^{1} \mathrm{H}_{2} \mathrm{O}$ (Milli-Q quality) and $10 \mathrm{wt} \%{ }^{2} \mathrm{H}_{2} \mathrm{O}\left(99.8 \mathrm{~mol} \%{ }^{2} \mathrm{H}\right.$, Armar Chemicals, Switzerland) in order to allow for NMR observation of both ${ }^{1} \mathrm{H}$ and ${ }^{2} \mathrm{H}$ nuclei. All other chemicals were of analytical grade and purchased from Sigma-Aldrich, Sweden. The samples were prepared by weighing appropriate amounts of the ingredients into $10 \mathrm{ml}$ vials, which were sealed by screw caps and centrifuged until the mixtures turned homogeneous. Subsequently, $0.5 \mathrm{ml}$ was transferred to $5 \mathrm{~mm}$ disposable NMR tubes, which were flame sealed and subjected to further centrifugation to remove air bubbles. One $\mathrm{C}_{14} \mathrm{E}_{5}$ sample (61.2 wt $\%$ surfactant) underwent temperature cycling within the field of the NMR magnet as described by Bernin et al. ${ }^{27}$ in order to produce an $\mathrm{L}_{\alpha}$ phase with lamellar directors aligned with the surface normals of the tube walls, while another $\mathrm{C}_{14} \mathrm{E}_{5}$ sample (59.7 wt\% surfactant) was not exposed to any further treatment after centrifugation of the NMR tube. These two samples will be referred to as "oriented $\mathrm{L}_{\alpha}$ " and "random $\mathrm{L}_{\alpha}$ ", respectively. NMR experiments on the $\mathrm{C}_{14} \mathrm{E}_{5}$ samples were performed at $50 \pm 1{ }^{\circ} \mathrm{C}$. The $\mathrm{AOT} /$ isooctane/water samples were melted to reverse micellar phases at $50{ }^{\circ} \mathrm{C}$, and subsequently cooled down at different rates to produce varying degrees of domain alignment. ${ }^{1,78}$ One sample (38 wt\% AOT, $14 \mathrm{wt} \%$ isooctane) was slowly cooled to $20{ }^{\circ} \mathrm{C}$ over $12 \mathrm{~h}$, giving domains preferentially aligned perpendicular to the surface normals of the tube walls. The sample was further equilibrated at $20{ }^{\circ} \mathrm{C}$ for 12 months before the NMR experiments. A second sample (44 wt\% AOT, $17 \mathrm{wt} \%$ isooctane) was cooled to $15{ }^{\circ} \mathrm{C}$ in less than $1 \mathrm{~min}$, giving nearly randomly oriented domains, and was immediately investigated with NMR. These samples will be referred to as "oriented $\mathrm{H}_{\mathrm{II}}$ " and "random $\mathrm{H}_{\mathrm{II}}$ ", respectively. The $\mathrm{H}_{\text {II }}$ samples were studied with NMR at $20 \pm 1{ }^{\circ} \mathrm{C}$.

\section{NMR experiments}

NMR experiments were carried out on a Bruker Avance II 500 spectrometer (Bruker, Karlsruhe, Germany) operating at 500.13 MHz ${ }^{1} \mathrm{H}$ resonance frequency. The $11.7 \mathrm{~T}$ magnet was equipped with a MIC-5 probe capable of delivering $3 \mathrm{~T} \mathrm{~m}^{-1}$ magnetic field gradients in three orthogonal directions. The sample temperature was controlled with a stream of air using a BVT 2000 unit. Diffusion MRI experiments were performed with Topspin 2.1 using the pulse sequence in Fig. 1(a), which is based on the sequence introduced by Lasič et al. ${ }^{50}$ The images were read out with a rapid acquisition with relaxation enhancement (RARE) block, ${ }^{79}$ giving $4.8 \times 4.8 \mathrm{~mm}$ field-of-view $(x \times y)$, $128 \times 32$ acquisition matrix size, $5 \mathrm{~mm}$ slice thickness $(z)$, and 65 ms duration of the echo train. The relation between the imaging slice and the NMR tube is shown in Fig. 2. The RARE block was

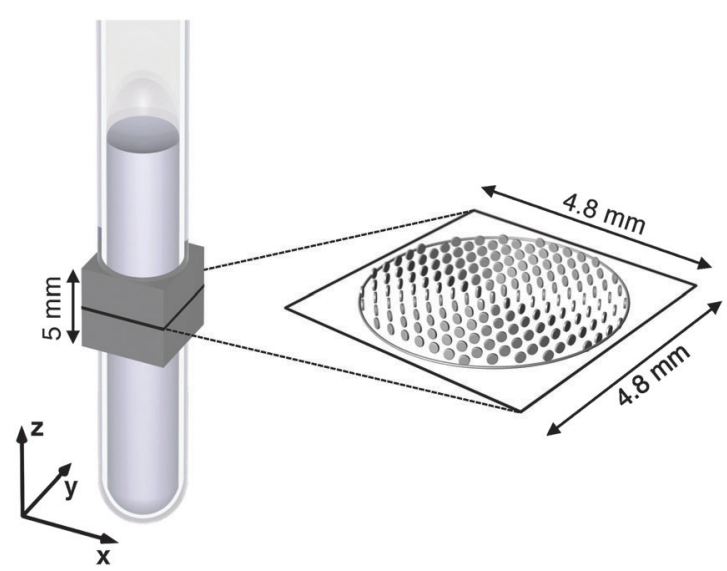

Fig. 2 Schematic geometry for the MRI experiments on samples with $0.5 \mathrm{ml}$ liquid crystal in an NMR tube with $5 \mathrm{~mm}$ outer diameter. The coils for generating the magnetic field gradients define the $x y z$ lab frame. The gray box indicates the $5 \mathrm{~mm}$ thick slice excited in the MRI experiments. The magnified black square shows the $4.8 \times 4.8 \mathrm{~mm}$ field-of-view of the image plane, while the gray circle delineates the outer surface of the liquid crystal within this plane. The tensor glyphs represent voxel-average diffusion tensors $\langle\mathbf{D}\rangle$ for the oriented $L_{\alpha}$ sample obtained at $0.3 \mathrm{~mm} \times 0.3 \mathrm{~mm}$ resolution in the $x y$-plane. 
preceded by a spin-echo diffusion-encoding block with $35 \mathrm{~ms}$ duration. Identical gradient waveforms $\mathbf{G}(t)$ of duration $\tau=15.9 \mathrm{~ms}$ and maximum gradient amplitude of approximately $1.2 \mathrm{~T} \mathrm{~m}^{-1}$ were located on each side of the $180^{\circ}$ pulse. A shaped gradient pulse with $1.59 \mathrm{~ms}$ quarter-sine ramp-up and $2.39 \mathrm{~ms}$ half-cosine ramp-down was used to define an axial waveform $G_{\mathrm{A}}(t)$, which was converted to the radial waveform $G_{\mathrm{R}}(t)$ and $\mathbf{G}(t)$ as described with the scheme in Fig. 1(b). The diffusion-encoding tensor $\mathbf{b}$ was sampled for a grid of four magnitudes $b$ (geometric spacing from 26 to $8610 \times 10^{6} \mathrm{~s} \mathrm{~m}^{-2}$ ), four anisotropies $b_{\Delta}$ (linear spacing from -0.5 to 1$)$, and 31 directions $(\Theta, \Phi)$ chosen according to the electrostatic repulsion scheme, ${ }^{80}$ giving in total 496 images. The values of $b_{\Delta}$ and $b$ were varied by changing the angle $\zeta$ and scaling the amplitudes of the waveforms $G_{\mathrm{A}}(t)$ and $G_{\mathrm{R}}(t)$ at constant timing parameters. Each image was acquired as the sum of two transients at $3 \mathrm{~s}$ recycle delay, resulting in $52 \mathrm{~min}$ of total experiment time.

\section{Data analysis}

All data processing was performed with in-house code written in Matlab (MathWorks, Natick, MA). The images were reconstructed at $128 \times 128$ matrix size, giving $37.5 \mu \mathrm{m} \times 37.5 \mu \mathrm{m}$ nominal spatial resolution, and subjected to $0.15 \mathrm{~mm}$ Gaussian smoothing. For each voxel, the average diffusion tensor $\langle\mathbf{D}\rangle$ was evaluated by non-linear fitting of eqn (19) to the acquired signal intensities $I(\mathbf{b}),{ }^{72,73}$ using the initial intensity $I_{0}$ and the three eigenvalues and Euler angles of $\langle\mathbf{D}\rangle$ as adjustable parameters. Equations for evaluating the tensor elements $\left\langle D_{i j}\right\rangle$ from the eigenvalues and Euler angles can be found in, e.g., the tutorial by Kingsley, ${ }^{81}$ while eqn (25) gives the relations between the $\mathbf{b}$ tensor elements and its parameterization as $b, b_{\Delta}, \Theta$, and $\Phi$. The sizes and shapes of the microscopic diffusion tensors $\mathbf{D}$ were estimated on a voxel-by-voxel basis by averaging the $I(\mathbf{b})$ data over the 31 acquisition directions, ${ }^{50}$ leaving a reduced set of $4 \times 4$ data points $I\left(b, b_{\Delta}\right)$, and fitting eqn (29) to the data using $I_{0}, D_{\text {iso }}$, and $D_{\Delta}$ as adjustable parameters. ${ }^{60}$ Subsequently, the elements of the Saupe order tensor $\mathbf{S}$ were evaluated by inserting the values of $\left\langle D_{i j}\right\rangle, D_{\text {iso }}$, and $D_{\Delta}$ into eqn (12). The fit results were downsampled to $16 \times 16$ matrix size, corresponding to $0.3 \mathrm{~mm} \times 0.3 \mathrm{~mm}$ spatial resolution, when displaying the results as superquadric tensor glyphs, ${ }^{71}$ while the $128 \times 128$ matrix size was used for generating color-coded parameter maps.

\section{Results and discussion}

Experimental tensors $\langle\mathbf{D}\rangle, \mathbf{D}$, and $\mathbf{S}^{\prime}$ are shown in Fig. 3 for all samples. The oriented $\mathrm{L}_{\alpha}$ sample features oblate $\left(\lambda_{\Delta}<0\right)$ tensors $\langle\mathbf{D}\rangle$ with minor axes in parallel with the normal vectors of the tube wall. The nearly planar shape $\left(\lambda_{\Delta}=-1 / 2\right)$ is consistent
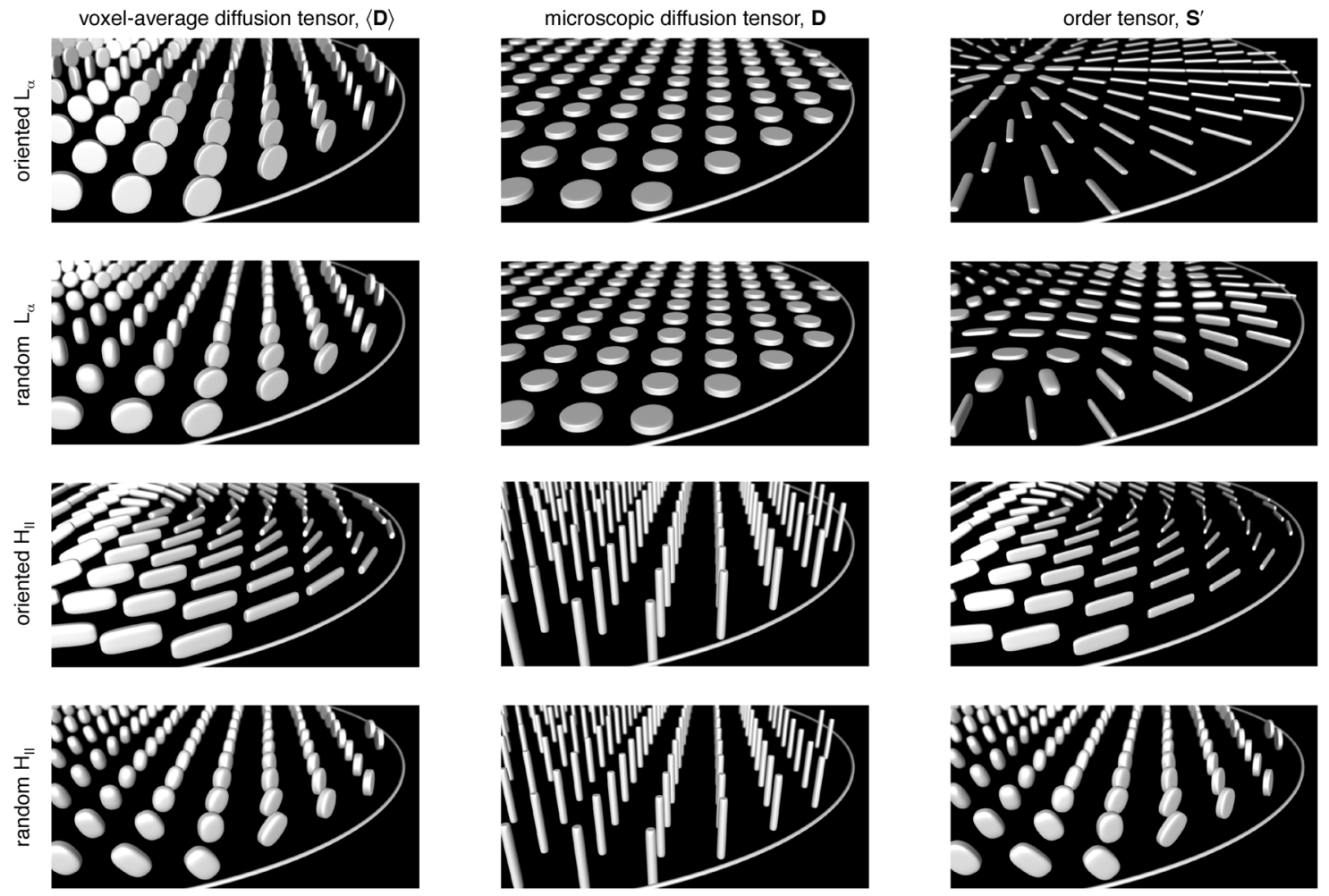

Fig. 3 Experimental results for the oriented $L_{\alpha}$ (row 1), random $L_{\alpha}$ (row 2), oriented $H_{\|}$(row 3), and random $H_{\|}$(row 4) samples displayed as superquadric tensor glyphs representing the voxel-average diffusion tensor $\langle\mathbf{D}\rangle$ (column 1), microscopic diffusion tensor $\mathbf{D}$ (column 2), and order tensor $\mathbf{S}^{\prime}$ (column 3 ) at a spatial resolution of $0.3 \mathrm{~mm} \times 0.3 \mathrm{~mm}$ in the $x y$-plane. The figures show an oblique view of a section of the image plane illustrated in Fig. 2 . The experiment for determining $\mathbf{D}$ is designed to be insensitive to tensor orientation, and, for simplicity, all these tensors are shown with the cylindrical symmetry axis along the $z$-direction. 
with the underlying lamellar geometry of the liquid crystal, but could according to eqn (13) in principle result from an ensemble of linear microscopic diffusion tensors $\left(D_{\Delta}=1\right)$ randomly oriented within a single plane $\left(S_{Z Z}=-1 / 2\right)$. All experimental $\mathbf{D}$ are oblate with values of the $D_{\Delta}$ around -0.49 , proving that the liquid crystal is of the lamellar type throughout the sample. Using the knowledge of the size and shape of $\mathbf{D}$, information about the director orientations can be disentangled from $\langle\mathbf{D}\rangle$ using eqn (12). For nearly all voxels of the oriented $\mathrm{L}_{\alpha}$ sample, the order tensors $\mathbf{S}^{\prime}$ are linear $\left(S_{Z Z} \approx 1\right)$, indicating a single preferred director orientation within each voxel, and oriented radially with respect to the tube axis. A few exceptions occur in the very center of the tube, where the planar shapes of the $\mathbf{S}^{\prime}$ tensors show that the voxels contain a distribution of director orientations within the $x y$-plane.

The $\langle\mathbf{D}\rangle$ tensors for the random $\mathrm{L}_{\alpha}$ sample have shapes ranging from oblate $\left(\lambda_{\Delta}<0\right)$ to prolate $\left(\lambda_{\Delta}>0\right)$. Since all $\mathbf{D}$ tensors are identical also in this case, the varying shapes of $\langle\mathbf{D}\rangle$ result from the voxel-scale orientation distributions rather than from any differences in the microscopic geometries of the liquid crystal. The resulting $\mathbf{S}^{\prime}$ tensors cover a range of shapes, but they are all mainly located within the $x y$-plane, indicating that the directors in the "random" $L_{\alpha}$ sample tend to avoid the $z$-direction just as for the oriented $\mathrm{L}_{\alpha}$ sample, albeit with a less distinct radial pattern.

The oriented $\mathrm{H}_{\text {II }}$ sample yields mainly prolate $\langle\mathbf{D}\rangle$ tensors in a pattern forming nearly concentric circles in the image plane, while the random $\mathrm{H}_{\mathrm{II}}$ sample features $\langle\mathbf{D}\rangle$ tensors covering a range of shapes from oblate to spherical. The corresponding $\mathbf{D}$ tensors all have prolate shapes and values of $D_{\Delta}$ around 0.9 , leading to similar shapes and identical semi-axis orientations of the $\langle\mathbf{D}\rangle$ and $\mathbf{S}^{\prime}$ tensors. The concentric pattern of $\langle\mathbf{D}\rangle$ tensor orientations for the oriented $\mathrm{H}_{\mathrm{II}}$ sample thus directly corresponds to the pattern of director orientations. The nearly spherical $\langle\mathbf{D}\rangle$ and $\mathbf{S}^{\prime}$ tensors in the interior of the random $\mathrm{H}_{\mathrm{II}}$ sample verify that the directors to a reasonable approximation are randomly oriented, while the more oblate tensors close to the glass surface indicate that director orientations in parallel with the surface normal vectors are less favorable.

In order to more clearly visualize the shapes and orientations of the $\mathbf{S}^{\prime}$ tensors throughout the entire image plane, colorcoded maps were generated as shown and explained in Fig. 4. The oriented $\mathrm{L}_{\alpha}$ sample has perfect radial director orientations throughout the sample (red and green colors at surfaces with normal vectors along $x$ and $y$, respectively). The pattern with director orientations in concentric circles (green and red at normal vectors along $x$ and $y$, respectively) is well developed for most parts of the oriented $\mathrm{H}_{\text {II }}$ sample, but somewhat less distinct in the third quadrant of the image where the bluish tint hints at directors aligned with the tube axis. Both "random" samples feature irregular patterns and color variations on multiple length scales. Also for these samples the director orientations are clearly affected by the presence of the tube walls: the random $\mathrm{L}_{\alpha}$ sample shows a rim of radially oriented directors, while the directors avoid the radial orientation in the random $\mathrm{H}_{\mathrm{II}}$ sample as evidenced by the dominance of turquoise and purple colors close to surfaces with normal vectors in the $x$ - and $y$-directions, respectively.
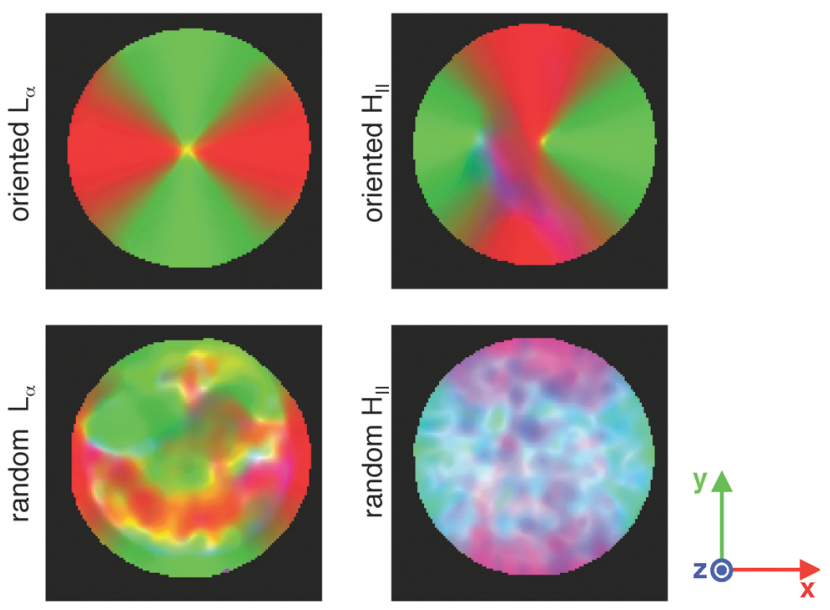

Fig. 4 Maps of the order tensor $\mathbf{S}^{\prime}$ with color-coding according to $[R, G, B]=\left[S_{x x}{ }^{\prime}, S_{y y}{ }^{\prime}, S_{z z}{ }^{\prime}\right] / S_{33^{\prime}}$, where $S_{33^{\prime}}$ is the largest eigenvalue of $\mathbf{S}^{\prime}$. Linear tensors give red, green, and blue colors when they are oriented in, respectively, the $x^{-}, y^{-}$, and, $z$-directions. The colors of planar tensors are obtained by additive color mixing: yellow (red + green) for tensors spanning the $x y$-plane, purple (red + blue) for the $x z$-plane, and turquoise (green + blue) for the $y z$-plane. White (red + green + blue) corresponds to spherical tensors. The field-of-view is $4.8 \mathrm{~mm} \times 4.8 \mathrm{~mm}$ in the $x y$-plane.

\section{Conclusions}

We have shown that information from standard diffusion tensor imaging and our recent method for measuring the size and shape of microscopic diffusion tensors can be combined to generate fields of Saupe order tensors, thereby enabling quantification of liquid crystal director orientations at an unprecedented level of detail. By defining a shifted and rescaled order tensor, the spatial pattern of director orientations were visualized as arrays of tensor glyphs and color-coded maps. In addition to detailed multi-scale characterization of liquid crystalline materials, we believe that our approach can be adapted to in vivo studies of cell shapes and orientations in normal and pathological tissues by minor modifications of the pulse sequence used by Szczepankiewicz et al. ${ }^{69}$

\section{Acknowledgements}

We thank Patrick Offer (RWTH Aachen University, Germany) and Karin Bryskhe (CR Development, Lund, Sweden) for preparing the liquid crystal samples, and Dan Lundberg (CR Competence, Lund, Sweden) for suggesting the composition of the reverse hexagonal phase. This work was financially supported by the Swedish Foundation for Strategic Research (AM13-0090) and the Swedish Research Council (2014-3910). DT is co-owner of the company CR Development AB (Lund, Sweden), holding patents related to the described method.

\section{References}

1 G. J. T. Tiddy, Phys. Rep., 1980, 57, 1-46.

2 B. Jönsson, B. Lindman, K. Holmberg and B. Kronberg, Surfactants and Polymers in Aqueous Solution, John Wiley \& Sons Ltd, Chichester, 1998. 
3 D. F. Evans and H. Wennerström, The Colloidal Domain: Where Physics, Chemistry, Biology, and Technology Meet, Wiley-VCH, New York, 2nd edn, 1999.

4 C. Beaulieu, NMR Biomed., 2002, 15, 435-455.

5 P. G. de Gennes and J. Prost, The Physics of Liquid Crystals, Clarendon Press, Oxford, 2nd edn, 1995.

6 K. G. Götz and K. Heckmann, J. Colloid Sci., 1958, 13, 266-272.

7 K. D. Lawson and T. J. Flautt, J. Am. Chem. Soc., 1969, 89, 5489-5491.

8 K. Radley, L. W. Reeves and A. S. Tracey, J. Phys. Chem., 1976, 80, 174-182.

9 B. J. Forrest and L. W. Reeves, Chem. Rev., 1981, 81, 1-14.

10 N. Boden, S. A. Corne and K. W. Jolley, Chem. Phys. Lett., 1984, 105, 99-103.

11 P. J. Photinos and A. Saupe, J. Chem. Phys., 1986, 84, 517-521.

12 P. J. Photinos and A. Saupe, J. Chem. Phys., 1986, 85, 7467-7471.

13 J. Chung and J. H. Prestegard, J. Phys. Chem., 1993, 97, 9837-9843.

14 G. Briganti, A. L. Segre, D. Capitani, C. Casieri and C. La Mesa, J. Phys. Chem. B, 1999, 103, 825-830.

15 D. Capitani, C. Casieri, G. Briganti, C. La Mesa and A. L. Segre, J. Phys. Chem. B, 1999, 103, 6088-6095.

16 T. D. Le, U. Olsson, K. Mortensen, J. Zipfel and W. Richtering, Langmuir, 2001, 17, 999-1008.

17 F. Nettesheim, I. Grillo, P. Lindner and W. Richtering, Langmuir, 2004, 20, 3947-3953.

18 A. Yethiraj, D. Capitani, N. E. Burlinson and E. E. Burnell, Langmuir, 2005, 21, 3311-3321.

19 J. S. Clawson, G. P. Holland and T. M. Alam, Phys. Chem. Chem. Phys., 2006, 8, 2635-2641.

20 Y. Iwashita and H. Tanaka, Nat. Mater., 2006, 5, 147-152.

21 D. Capitani, A. Yethiraj and E. E. Burnell, Langmuir, 2007, 23, 3036-3048.

22 T. M. Alam and S. K. McIntyre, Langmuir, 2008, 24, 13890-13896.

23 B. Medronho, S. Shafei, R. Szopko, M. G. Miguel, U. Olsson and C. Schmidt, Langmuir, 2008, 24, 6480-6486.

24 B. Medronho, J. Brown, M. G. Miguel, C. Schmidt, U. Olsson and P. Galvosas, Soft Matter, 2011, 7, 4938-4947.

25 D. Sato, K. Obara, Y. Kawabata, M. Iwahashi and T. Kato, Langmuir, 2013, 29, 121-132.

26 B. Medronho, U. Olsson, C. Schmidt and P. Galvosas, Z. Phys. Chem., 2012, 226, 1293-1314.

27 D. Bernin, V. Koch, M. Nydén and D. Topgaard, PLoS One, 2014, 9, e98752.

28 O. Freund, J. Amédee, D. Roux and R. Laversanne, Life Sci., 2000, 67, 411-419.

29 F. Tiberg and M. Johnsson, J. Drug Delivery Sci. Technol., 2011, 21, 101-109.

30 G. S. Attard, J. C. Glyde and C. G. Göltner, Nature, 1995, 378, 366-368.

31 A. Firouzi, D. J. Schaefer, S. H. Tolbert, G. D. Stucky and B. F. Chmelka, J. Am. Chem. Soc., 1997, 119, 9466-9477.
32 A. Saupe and G. Englert, Phys. Rev. Lett., 1963, 11, 462-464. 33 Nuclear Magnetic Resonance of Liquid Crystals, ed. J. W. Emsley, D. Reidel Publishing Company, Dordrecht, 1985.

34 A. D. Rey, Soft Matter, 2010, 6, 3402-3429.

35 S. Kumar, J. D. Litster and C. Rosenblatt, Phys. Rev. A: At., Mol., Opt. Phys., 1983, 28, 1890-1892.

36 R. Moldovan and M. R. Puica, Phys. Lett. A, 2001, 286, 205-209.

37 A. Sonnet, A. Kilian and S. Hess, Phys. Rev. E: Stat. Phys., Plasmas, Fluids, Relat. Interdiscip. Top., 1995, 52, 718-722.

38 T. Tsuji and A. D. Rey, Phys. Rev. E: Stat. Phys., Plasmas, Fluids, Relat. Interdiscip. Top., 1998, 57, 5609-5625.

39 T. J. Jankun-Kelly and K. Mehta, IEEE Trans. Vis. Comput. Graph., 2006, 12, 1197-1204.

40 K. D. Lawson and T. J. Flautt, J. Phys. Chem., 1968, 72, 2066-2074.

41 R. Blinc, K. Easwaran, J. Pirš, M. Volfan and I. Zupančič, Phys. Rev. Lett., 1970, 25, 1327-1330.

42 N.-O. Persson, K. Fontell, B. Lindman and G. J. T. Tiddy, J. Colloid Interface Sci., 1975, 53, 461-466.

43 G. J. T. Tiddy, J. Chem. Soc., Faraday Trans. 1, 1977, 73, 1731-1737.

44 G. Chidichimo, L. Coppola, C. La Mesa, G. A. Ranieri and A. Saupe, Chem. Phys. Lett., 1988, 145, 85-89.

45 M. C. Holmes, P. Sotta, Y. Hendrikx and B. Deloche, J. Phys. II, 1993, 3, 1735-1746.

46 S. R. Wassall, Biophys. J., 1996, 71, 2724-2732.

47 H. Jóhanneson and B. Halle, J. Chem. Phys., 1996, 104, 6807-6817.

48 S. Gaemers and A. Bax, J. Am. Chem. Soc., 2001, 123, 12343-12352.

49 K. Szutkowski and S. Jurga, J. Phys. Chem. B, 2010, 114, 165-173.

50 S. Lasič, F. Szczepankiewicz, S. Eriksson, M. Nilsson and D. Topgaard, Front. Phys., 2014, 2, 11.

51 S. Funari, M. C. Holmes and G. J. T. Tiddy, J. Phys. Chem., 1992, 96, 11029-11038.

52 P. L. Hubbard, K. M. McGrath and P. T. Callaghan, J. Phys. Chem. B, 2006, 110, 20781-20788.

53 B. Medronho, C. Schmidt, U. Olsson and M. G. Miguel, Langmuir, 2010, 26, 1477-1481.

54 J. R. Brown and P. T. Callaghan, Soft Matter, 2011, 7, 10472-10482.

55 P. Trigo-Mourino, C. Merle, M. R. Koos, B. Luy and R. R. Gil, Chem. - Eur. J., 2013, 19, 7013-7019.

56 E. Fischer and P. T. Callaghan, Europhys. Lett., 2000, 50, 803-809.

57 S. Bulut, I. Åslund, D. Topgaard, H. Wennerström and U. Olsson, Soft Matter, 2010, 6, 4520-4527.

58 J. Winterhalter, D. Maier, D. A. Grabowski, J. Honerkamp, S. Müller and C. Schmidt, J. Chem. Phys., 1999, 110, 4035-4046.

59 P. J. Basser, J. Mattiello and D. Le Bihan, Biophys. J., 1994, 66, 259-267.

60 S. Eriksson, S. Lasič, M. Nilsson, C.-F. Westin and D. Topgaard, J. Chem. Phys., 2015, 142, 104201. 
61 C.-F. Westin, F. Szczepankiewicz, O. Pasternak, E. Özarslan, D. Topgaard, H. Knutsson and M. Nilsson, Med. Image Comput. Comput. Assist. Interv., 2014, 17, 209-216.

62 J. Sjölund, F. Szczepankiewicz, M. Nilsson, D. Topgaard, C.-F. Westin and H. Knutsson, J. Magn. Reson., 2015, 261, 157-168.

63 J. P. de Almeida Martins and D. Topgaard, Phys. Rev. Lett., 2016, 116, 087601.

64 C.-F. Westin, H. Knutsson, O. Pasternak, F. Szczepankiewicz, E. Özarslan, D. van Westen, C. Mattisson, M. Bogren, L. O'Donnell, M. Kubicki, D. Topgaard and M. Nilsson, NeuroImage, 2016, DOI: 10.1016/j.neuroimage.2016.02.039.

65 R. Blinc, M. Burgar, M. Luzar, J. Pirš, I. Zupančič and S. Žumer, Phys. Rev. Lett., 1974, 33, 1192-1195.

66 U. Hong, J. Kärger, R. Kramer, H. Pfeifer, G. Seiffert, U. Müller, K. K. Unger, H.-B. Lück and T. Ito, Zeolites, 1991, 11, 816-821.

67 H. Jóhannesson, I. Furó and B. Halle, Phys. Rev. E: Stat. Phys., Plasmas, Fluids, Relat. Interdiscip. Top., 1996, 53, 4904-4917.

68 T. M. Ferreira, D. Bernin and D. Topgaard, Annu. Rep. NMR Spectrosc., 2013, 79, 73-127.

69 F. Szczepankiewicz, S. Lasič, D. van Westen, P. C. Sundgren, E. Englund, C.-F. Westin, F. Ståhlberg, J. Lätt, D. Topgaard and M. Nilsson, NeuroImage, 2015, 104, 241-252.
70 D. Topgaard, Microporous Mesoporous Mater., 2013, 178, 60-63.

71 G. Kindlmann, Proceedings IEEE TVCG/EG Symposium on Visualization, 2004.

72 W. S. Price, NMR studies of translational motion, Cambridge University Press, Cambridge, 2009.

73 P. T. Callaghan, Translational dynamics \& magnetic resonance, Oxford University Press, Oxford, 2011.

74 S. Eriksson, S. Lasič and D. Topgaard, J. Magn. Reson., 2013, 226, 13-18.

75 R. F. Karlicek Jr and I. J. Lowe, J. Magn. Reson., 1980, 37, 75-91.

76 E. O. Stejskal and J. E. Tanner, J. Chem. Phys., 1965, 42, 288-292.

77 W. S. Price and P. W. Kuchel, J. Magn. Reson., 1991, 94, 133-139.

78 I. Åslund, C. Cabaleiro-Lago, O. Söderman and D. Topgaard, J. Phys. Chem. B, 2008, 112, 2782-2794.

79 J. Henning, A. Nauerth and H. Friedurg, Magn. Reson. Med., 1986, 3, 823-833.

80 M. Bak and N. C. Nielsen, J. Magn. Reson., 1997, 125, 132-139.

81 P. B. Kingsley, Concepts Magn. Reson., Part A, 2006, 28A, 101-122. 